\title{
A New Approach of Artificial Intelligence (AI) to Cyber Security
}

\author{
Kavita Srivastava \\ Faculty of BCA, Department of Business Management And Entrepreneurship, \\ Dr. R.M.L. A. University Ayodhya
}

\begin{abstract}
In this study, the concept of artificial intelligence and cyber security was evaluated in various terms, and its importance that contributes to illustrate as well. Based on the artificial intelligence, a significant security features which is widely used in artificial intelligence. Basic principles and parameters of artificial intelligence are directly affected to cyber security. In the Second Phase, how to significant of artificial intelligence affected to positive impacts on cyber security environment. Furthermore, we present a critical study of cyber security requests and analyses the views of recovering the cyber security capabilities by Artificial Intelligence theory.
\end{abstract}

Keywords: Artificial Intelligence, Cyber Security, Challenges

\section{INTRODUCTION}

Artificial Intelligence process is a critical task that involves a number of stages such as rule based systems, context awareness and retention, domain specific expertise. A most important challenge in Artificial Intelligence process is to advance fault detection at cyber security. The concept of Artificial Intelligence is closely related to cyber security [3]. Experts and Researchers are continuously concerning about Artificial Intelligence process, whose primary ambitions are to implement a artificial intelligence and cyber security. Cyber security means an unintended user are prevented to operate the system under any environment. Any positive effort to Cyber security is called an Artificial Intelligence process; it can be in complete solutions [4, 8]. Cyber security implements with cyber cell and produced complete security with a specific domains. This theoretical analysis is focuses on a set of specific parameters for highly security at cyber security issues. The three basic cyber security factors are namely confidentiality, integrity and availability $[7,10]$.

\section{ARTIFICIAL INTELLIGENCE}

After 50 years in the midst of which artificial intelligence (AI) has been a portrayed and dynamic field, there have been a couple of composing thinks about. At any rate the field is phenomenally difficult to exemplify either successively or explicitly. We suggest that the clarification behind this is there has never been a groundswell of effort inciting an apparent achievement. Never-the-less, there is a noteworthy gathering of composing which the learner must pro before attempting to consider what has ended up being a hydra-headed mammoth.
This audit endeavours to arrange the writing in a way which can be appreciated. Negatu, 2006 [1] taken a gander at the contrast between the negligible self and story self. The negligible self is just worried about what's going on at present, while, the story self requires recollections of the past and can get ready for what's to come.

Finland and P. Vogt, 2007 [2] believe it to be impossible to build consciousness. Their theory is that consciousness is a function of shared goals and social interaction. Therefore, at most, robots can only be extensions of humans and cannot act independently.

G. Bittencourt, 2007 [5] propose a model with six dimensions stacked over one another, where each dimension has an alternate arrangement of conduct capacities. Two feeling esteem criteria are utilized to make vertical and even conduct choice trying to amplify joy. A working PC recreation was created which functioned of course inside its essential condition.

J. G. Taylor, 1994 [6] propose a model with six dimensions stacked over one another, where each dimension has an alternate arrangement of conduct capacities. Two feeling esteem criteria are utilized to make vertical and even conduct choice trying to amplify joy. A working PC diversion was made which worked obviously inside its basiccondition. dimension of data preparing changed, in view of visual data being gotten. In his model, outside data preparing is led when the robot is wakeful. In any case, when the robot is in rest mode, outside data preparing is decreased and increasingly inside data having is led. 


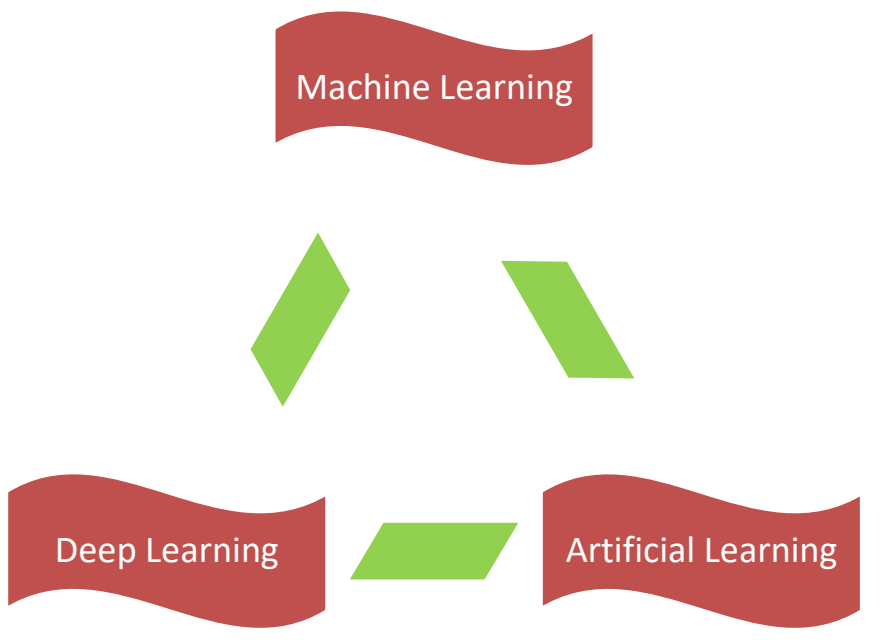

Figure 1 Artificial Intelligence Mode

Artificial intelligence is a part of computer science that aims to make smart machines. It has turned into a fundamental piece of the innovation business. Research related with artificial intelligence is exceptionally specialized and concentrated. The centre issues of artificial intelligence incorporate programming computers for certain traits [10].

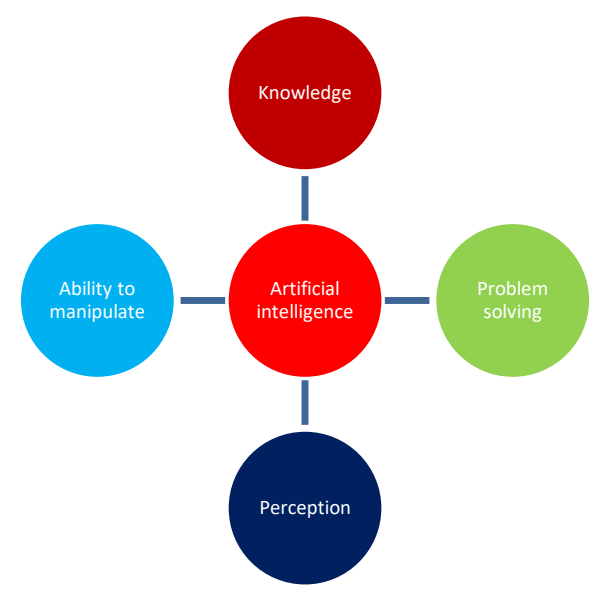

Figure 2 Programming Concepts of Artificial Intelligence

\section{CONCLUSION}

Artificial Intelligence process is a complex issue. Cyber security plays an important role in artificial intelligence process. This research paper studies the report on an artificial intelligence process and Cyber security which helps a developer to understand the Cyber security in Artificial Intelligence process. This paper concludes that Artificial Intelligence process depends on the parameters and it finally affects the Cyber security. Programming Concepts of Artificial Intelligence affects the Cyber security positively.

\section{REFERENCES}

[1] Negatu, S. D'Mello, and S. Franklin, "Cognitively inspired anticipation and anticipatory learning mechanisms for autonomous agents", Springer, vol. 4520, Berlin, pp. 108-127, 2006.

[2] P. Vogt, "Language evolution and robotics: issues on symbol grounding and language acquisition", in "Artificial Cognition Systems", A. Loula, R. Gudwin, and J. Queiroz (Editors), Hershey Printing and Publishing, Hershey, pp. 176-209, 2007.

[3] P. Grim and T. Kokalis, "Envromental variability and the emergence of meaning: simulated studies across imitation, genetic 


\section{Available online at www.ijrat.org}

algorithms, and neural networks," in "Artificial Cognition Systems", A. Loula, R. Gudwin, and J. Queiroz (Editors), Hershey Printing and Publishing, Hershey, pp. 284-326, 2007.

[4] D. Parisi and M. Mirolli, "Steps towards artificial consciousness: a robot's knowledge of its own body", American Association for Artificial Intelligence; Fall Symposium on Theoretical Fundations and current Approachs, Washington DC, November 2007.

[5] G. Bittencourt and J. Marchi, "An embodied logical model for cognition in artificial cognition systems", in "Artificial Cognition Systems", A. Loula, R. Gudwin, and J. Queiroz (Editors), Hershey Printing and Publishing, Hershey, 2007.

[6] J. G. Taylor, "Goals, drives, and consciouness", Neural Networks, vol. 7, pp. 1181-1190, 1994.

[7] J. Kivimaa, A. Ojamaa, E. Tyugu. Graded Security Expert System. Lecture Notes in
Computer Science, v.5508. Springer, 2009, 279-286.

[8] B. Iftikhar, A. S. Alghamdi, "Application of artificial neural network in detedction of dos attacks", in SIN 09: Proceedings of the $2^{\text {nd }}$ international conference on Security of information and networks. New York NY USA: ACM, 2009, pp.229-234.

[9] Kavita Srivastava, "A Critical Study of Cyber Security Challenges and Evaluation of Technology", International Journal of Modern Electronics and Communication Engineering (IJMECE) Volume No.-7, Issue No.-1, January, 2019.

[10] A. Mishra, D. Agarwal and M. H. Khan, "Availability Estimation Model: Fault Perspective", International Journal of Innovative Research in Science, Engineering and Technology, Vol. 6, Issue 6, June 2017. 